\title{
A PROBABILISTIC ANALYSIS OF THE MULTIKNAPSACK VALUE FUNCTION
}

\author{
M. MEANTI
}

Dipartimento di Matematica, University of Milano, Italy

\author{
A.H.G. RINNOOY KAN \\ Econometric Institute, Erasmus University, Rotterdam, The Netherlands

\section{STOUGIE} \\ Instituut voor Actuariaat en Econometrie, University of Amsterdam, The Netherlands \\ C. VERCELLIS \\ Dipartimento di Scienze del Informazione, University of Milano, Italy
}

Received 22 June 1987

Revised manuscript received 19 August 1988

The optimal solution value of the multiknapsack problem as a function of the knapsack capacities is studied under the assumption that the profit and weight coefficients are generated by an appropriate random mechanism. A strong asymptotic characterization is obtained, that yiclds a closed form expression for certain special cases.

Key words: Multiknapsack value function, Lagrangean relaxation, asymptotic characterization, uniform strong law of large numbers.

\section{Introduction}

The multiknapsack (MK) problem is to select a collection of items that yields maximal profit subject to a number of capacity constraints. More precisely, let us suppose that item $j(j=1, \ldots, n)$ requires $a_{i j}$ units of space in the $i$ th knapsack $(i=1, \ldots, m)$ and yields $c_{j}$ units of profit upon inclusion, and that the $i$ th knapsack has capacity $b_{i}(i=1, \ldots, m)$. Then the multiknapsack problem can be written as:

$$
\begin{array}{ll}
(\mathrm{MK}) \quad \max & \sum_{j=1}^{n} c_{j} x_{j} \\
\text { s.t. } & \sum_{j=1}^{n} a_{i j} x_{j} \leqslant b_{i} \quad(i=1,2, \ldots, m), \\
& x_{j} \in\{0,1\} \quad(j=1,2, \ldots, n) .
\end{array}
$$

This research was partially supported by NSF Grant ECS-83-16224, and MPI Project "Matematica computazionale". 
MK has been used to model problems in the areas of scheduling and capital budgeting [9]. The problem is known to be NP-hard [6]; it is a generalization of the standard knapsack problem $(m=1)$. MK can be solved by a polynomial approximation scheme [4], but a fully polynomial one cannot exist unless $P=$ NP [5].

In this paper, we are interested in the behavior of the solution value of $M K$ with respect to changing knapsack capacities. We shall show that if the coefficients $c_{j}$ and $a_{i j}(j=1, \ldots, n, i=1, \ldots, m)$ are generated by an appropriate random mechanism, then the sequence of optimal values, properly normalized, converges with probability one (w.p. 1) to a function of the $b_{i}$ 's, as $n$ goes to infinity and $m$ remains fixed. The function will be computed in closed form for some special cases.

A number of probabilistic analyses of algorithms for the single knapsack problem $(m=1)$ has been carried out in the past $[1,2,7,10]$, but the solution value has not yet been asymptotically characterized in the above fashion. A similar comment applies to the probabilistic analysis in [4] for MK under a stochastic model less general than the one considered here. Random variables will be indicated by boldface characters.

\section{Upper and lower bounds}

Let us assume that the profit coefficients $\boldsymbol{c}_{1}, \boldsymbol{c}_{2}, \ldots$, are nonnegative independent identically distributed (i.i.d.) random variables with common distribution function $F$ and finite expectation $E c_{1}$. Let $a_{j}=\left(a_{1 j}, a_{2 j}, \ldots, a_{m j}\right)$ for $j=1,2, \ldots$ We assume that $a_{1}, a_{2}, \ldots$, are nonnegative i.i.d. random vectors. Moreover, the profit coefficients and the requirement coefficients with different indices are assumed to be independent of each other.

We shall use the independent model approach for the sampling of a series of problem instances of growing size with the number $m$ of restrictions held fixed. Thus, each successive problem instance is drawn independently from the other problem instances. This is in contrast with the incremental model, in which a problem instance of size $n$ is built up from the coefficients of the problem instance of size $n-1$ by adding an extra set of coefficients $\left(\boldsymbol{c}_{n}, \boldsymbol{a}_{n}\right)$ that is drawn independently from the previous ones. We refer to [12] for an overview of the relation between these models. In particular, it is shown that convergence results in the independent model imply convergence results in the incremental model, thus making the independent model the more general of the two.

It is reasonable to assume that the capacities $b_{i}$ grow proportionally with the number of items. Specifically, let $b_{i}=n \beta_{i} \quad(i=1,2, \ldots, m)$ for $\beta \in V:=$ $\left\{\beta \mid 0<\beta<E \boldsymbol{a}_{1}\right\}$. As remarked in [10], the $i$ th constraint would tend to be redundant if $\beta_{i}>E a_{i 1}$, in the sense that it would asymptotically be satisfied with probability 1 even if all items were included.

We define $\boldsymbol{z}_{n}^{\mathrm{I}}$ to be the optimal solution value of MK and $\boldsymbol{z}_{n}^{\mathrm{LP}}$ to be the value of the LP-relaxation MKLP. Let $c_{\max }=\max _{j=1, \ldots, n}\left\{c_{j}\right\}$. 
Lemma 2.1. For every realization $\left(c_{1}, a_{1}\right),\left(c_{2}, a_{2}\right), \ldots,\left(c_{n}, a_{n}\right)$ of $\left(c_{1}, a_{1}\right)$, $\left(c_{2}, a_{2}\right), \ldots,\left(c_{n}, a_{n}\right)$, we have

$$
z_{n}^{\mathrm{LP}} \geqslant z_{n}^{\mathrm{I}} \geqslant z_{n}^{\mathrm{LP}}-m c_{\max } .
$$

Proof. The first inequality is trivial. An optimal basic solution of MKLP has at most $m$ basic variables that are fractional. Rounding down these values yields a feasible integer solution whose value is given by $z_{n}^{\mathrm{LP}}$ decreased by at most $m c_{\max }$.

Division of (2.1) by $n$ yields

$$
z_{n}^{\mathrm{LP}} / n \geqslant z_{n}^{\mathrm{I}} / n \geqslant z_{n}^{\mathrm{LP}} / n-m c_{\max } / n .
$$

Since, $E c_{1}<\infty$, we have that $\lim _{n \rightarrow \infty} c_{\max } / n=0$ w.p. 1. From these two observations asymptotic equivalence of $z_{n}^{\mathrm{I}} / n$ and $z_{n}^{\mathrm{LP}} / n$ w.p. 1 follows easily.

\section{Lemma 2.2.}

$$
\lim _{n \rightarrow \infty}\left|\frac{z_{n}^{\mathrm{I}}}{n}-\frac{z_{n}^{\mathrm{LP}}}{n}\right|=0 \quad \text { w.p. } 1
$$

\section{Asymptotic characterization of the solution value}

In order to derive a function of $b_{i}(i=1,2, \ldots, m)$ to which $z_{n}^{\mathrm{LP}}$ and hence $z_{n}^{\prime}$ is asymptotic w.p. 1, we consider the Lagrangean relaxation of MKLP, defined by

$$
\boldsymbol{w}_{n}(\lambda):=\max \left\{\sum_{i=1}^{m} \lambda_{i} b_{i}+\sum_{j=1}^{n}\left(c_{j}-\sum_{i=1}^{m} \lambda_{i} \boldsymbol{a}_{i j}\right) x_{j} \mid 0 \leqslant x_{j} \leqslant 1(j=1,2, \ldots, n)\right\},
$$

where $\lambda=\left(\lambda_{1}, \ldots, \lambda_{m}\right)$.

For every realization of the stochastic parameters, the function $w_{n}(\lambda)$ is convex over the region defined by $\lambda \geqslant 0$. Moreover,

$$
\min _{\lambda \geqslant 0} w_{n}(\lambda)=z_{n}^{\mathrm{LP}}
$$

If we define the random variables

$$
\boldsymbol{x}_{j}^{\mathbf{L}}(\lambda):=\left\{\begin{array}{l}
1 \quad \text { if } \boldsymbol{c}_{j}-\sum_{j=1}^{m} \lambda_{i} \boldsymbol{a}_{i j}>0, \quad(j=1,2, \ldots n), \\
0 \text { otherwise, }
\end{array}\right.
$$

then

$$
\boldsymbol{w}_{n}(\lambda)=\sum_{i=1}^{m} \lambda_{i} b_{i}+\sum_{j=1}^{n}\left(c_{j}-\sum_{i=1}^{m} \lambda_{i} \boldsymbol{a}_{i j}\right) \boldsymbol{x}_{j}^{\mathrm{l}}(\lambda)
$$


We also define $L_{n}(\lambda):=\boldsymbol{w}_{n}(\lambda) / n$, and for $c \in \mathbb{R}, a \in \mathbb{R}^{m}$ the function $f_{\lambda}: \mathbb{R}^{m+1} \rightarrow \mathbb{R}$ for any $\lambda \in \mathbb{R}^{m}$, as

$$
f_{\lambda}(c, a)=\lambda^{\mathrm{T}} \beta+\left(c-\lambda^{\mathrm{T}} a\right) x^{\mathrm{L}}(\lambda),
$$

with

$$
x^{\mathrm{L}}(\lambda)= \begin{cases}1 & \text { if } c>\lambda^{\mathrm{T}} a \\ 0 & \text { otherwise }\end{cases}
$$

Then $\boldsymbol{L}_{n}(\lambda)$ can be viewed as the mean value of the random variable $f_{\lambda}(\boldsymbol{c}, \boldsymbol{a})$ over $n$ independent observations $\left(c_{1}, a_{1}\right),\left(c_{2}, a_{2}\right), \ldots,\left(c_{n}, a_{n}\right)$. Because of the independence of $\left(c_{1}, a_{1}\right),\left(c_{2}, a_{2}\right), \ldots,\left(c_{n}, a_{n}\right)$, the random variables $x_{1}^{\mathrm{L}}(\lambda), x_{2}^{\mathrm{L}}(\lambda), \ldots, x_{n}^{\mathrm{L}}(\lambda)$ are also independent. Hence, the strong law of large numbers $[8,17.3]$ implies that, for every $\lambda \geqslant 0, L_{n}(\lambda)$ converges with probability 1 to the expectation

$$
E f_{\lambda}(\boldsymbol{c}, \boldsymbol{a})=\lambda^{\mathrm{T}} \beta+E \boldsymbol{c}_{1} \boldsymbol{x}_{1}^{\mathrm{L}}(\lambda)-\lambda^{\mathrm{T}} E \boldsymbol{a}_{1} \boldsymbol{x}_{1}^{\mathrm{L}}(\lambda) .
$$

Let us write $L(\lambda):=E f_{\lambda}(\boldsymbol{c}, \boldsymbol{a})$ and let $\lambda^{*}$ be a minimizer of $L(\lambda)$.

Let $\lambda_{n}^{*}$ be a vector of multipliers minimizing $w_{n}(\lambda)$. We shall now prove that $\boldsymbol{L}_{n}\left(\boldsymbol{\lambda}_{n}^{*}\right) \rightarrow L\left(\lambda^{*}\right)$ with probability 1 by first strengthening our previous result from pointwise to uniform convergence with probability 1 . Since the functions $L_{n}(\lambda)$ are convex for every realization of the stochastic parameters we may apply Theorem 10.8 from $[11$, p. 90$]$, which states that pointwise convergence of a sequence of convex functions on any compact subset of their domain implies uniform convergence on this subset to a function that is also convex. Hence, all we have to show is that a minimizing sequence $\left\{\boldsymbol{\lambda}_{n}^{*}\right\}(n=1,2, \ldots)$ and $\lambda^{*}$ are contained in a compact subset of $\mathbb{R}_{+}^{m}$.

Lemma 3.1. There exists a number $n_{1}$ such that for all $n \geqslant n_{1}, \boldsymbol{L}_{n}(\lambda)$ attains its minimum within the set

$$
S:=\left\{\lambda \mid \lambda \geqslant 0, \lambda^{\mathrm{T}} \beta \leqslant E c_{1}+1\right\}
$$

with probability $1 . L(\lambda)$ also attains its minimum value within $S$.

Proof. As a result of the strong law of large numbers we have that

$$
\operatorname{Pr}\left\{\exists n_{1}: \forall n \geqslant n_{1}, E c_{1}+1 \geqslant \frac{1}{n} \sum_{j=1}^{n} \boldsymbol{c}_{j}=L_{n}(0)\right\}=1 \text {. }
$$

Since $\boldsymbol{L}_{n}(\lambda) \geqslant \lambda^{\mathrm{T}} \beta$, we have for any $\lambda \notin S, \lambda \geqslant 0$, that $\boldsymbol{L}_{n}(\lambda)>E \boldsymbol{c}_{1}+1$. This together with the above probability statement yields that for any $\lambda \notin S$,

$$
\operatorname{Pr}\left\{\exists n_{1}: \forall n \geqslant n_{1}, L_{n}(\lambda) \geqslant E c_{1}+1 \geqslant L_{n}(0)\right\}=1 .
$$

Thus, for all $n \geqslant n_{1}, \lambda_{n}^{*} \in S$ with probability 1 . For $L(\lambda)$ we have that $L(\lambda) \geqslant \lambda^{\mathrm{T}} \beta>$ $E c_{1}=L(0)$ for $\lambda$ such that $\lambda^{\mathrm{T}} \beta>E c_{1}+1$, so that $\lambda^{*} \in S$ as well. 
Since we assumed that $E c_{1}$ exists, $S$ is indeed a compact set. We therefore have the following lemma.

Lemma $3.2\left[11\right.$, Theorem 10.8]. $L_{n}(\lambda) \rightarrow L(\lambda)$ w.p. 1 uniformly on $S . \quad \square$

We are now in a position to prove the required result.

Theorem 3.1. $\boldsymbol{L}_{n}\left(\boldsymbol{\lambda}_{n}^{*}\right) \rightarrow L\left(\lambda^{*}\right)$ w.p. 1 .

Proof. Uniform convergence w.p. 1 on $S$ can be written as

$$
\operatorname{Pr}\left\{\forall \varepsilon>0 \exists n_{0}: \forall n \geqslant n_{0}, \sup _{\lambda \in S}\left|\boldsymbol{L}_{n}(\lambda)-L(\lambda)\right|<\varepsilon\right\}=1 .
$$

If $\boldsymbol{L}_{n}\left(\boldsymbol{\lambda}_{n}^{*}\right)>L\left(\lambda^{*}\right)$, then $\left|\boldsymbol{L}_{n}\left(\lambda_{n}^{*}\right)-L\left(\lambda^{*}\right)\right| \leqslant \boldsymbol{L}_{n}\left(\lambda^{*}\right)-L\left(\lambda^{*}\right)$. A similar argument for the case that $\boldsymbol{L}_{n}\left(\boldsymbol{\lambda}_{n}^{*}\right)<L\left(\lambda^{*}\right)$ leads to the conclusion that

$$
\left|\boldsymbol{L}_{n}\left(\boldsymbol{\lambda}_{n}^{*}\right)-L\left(\lambda^{*}\right)\right| \leqslant \sup _{\lambda \in S}\left|\boldsymbol{L}_{n}(\lambda)-L(\lambda)\right| \text {. }
$$

Combination of (3.3) and (3.4) yields

$$
\operatorname{Pr}\left\{\forall \varepsilon>0 \exists n_{0}: \forall n \geqslant n_{0},\left|\boldsymbol{L}_{n}\left(\boldsymbol{\lambda}_{11}^{*}\right)-L\left(\lambda^{*}\right)\right|<\varepsilon\right\}=1 .
$$

Together (3.1), Theorem 3.1 and Lemma 2.2 imply the following result.

Theorem 3.2. $n^{-1} \boldsymbol{z}_{n}^{l} \rightarrow L\left(\lambda^{*}\right)$ w.p. 1 .

This theorem gives the required asymptotic characterization of the solution value of MK. $L\left(\lambda^{*}\right)$ is a function of the righthand sides $b_{i}(i=1,2, \ldots, m)$ and is defined implicitly by minimization of $L(\lambda)$ over $S$. Its explicit computation will be considered in Section 5. We note that the only assumptions that we made hitherto were the i.i.d. property of the stochastic parameters and the finiteness of the first moment of the profit coefficients. Under these assumptions also the following stronger version of Theorem 3.1 can be proved, indicating regular asymptotic behavior of the sequence of optimal Lagrangean multipliers $\boldsymbol{\lambda}_{n}^{*}$.

Theorem 3.3. With probability 1, the accumulation points of the series $\boldsymbol{\lambda}_{1}^{*}, \boldsymbol{\lambda}_{2}^{*}, \ldots$, are contained in the set of values of $\lambda$ that minimize $L(\lambda)$.

Proof. We consider the following inequality:

$$
\left|L\left(\boldsymbol{\lambda}_{n}^{*}\right)-L\left(\lambda^{*}\right)\right| \leqslant\left|L\left(\boldsymbol{\lambda}_{n}^{*}\right)-\boldsymbol{L}_{n}\left(\boldsymbol{\lambda}_{n}^{*}\right)\right|+\left|\boldsymbol{L}_{n}\left(\boldsymbol{\lambda}_{n}^{*}\right)-L\left(\lambda^{*}\right)\right| .
$$

Lemma 3.2 and Theorem 3.1 applied to the right-hand side imply immediately that $L\left(\boldsymbol{\lambda}_{n}^{*}\right) \rightarrow L\left(\lambda^{*}\right)$ w.p. 1. The proof can now be completed by application of Corollary 27.2 .1 in $[11$, p. 266]. 
In case $\lambda^{*}$ is the unique minimizer of $L(\lambda)$, we have that $\lambda_{n}^{*} \rightarrow \lambda^{*}$ w.p. 1 (see [11, Corollary 27.2 .2 , p. 266]).

\section{Smoothness properties}

In this section we investigate some properties of $L(\lambda)$ related to properties of the distributions of the stochastic parameters.

Recall the formulation (3.2) of $L(\lambda)$ and rewrite it as

$$
L(\lambda)=\lambda^{\mathrm{T}} \beta+E\left(\left(c_{1}-\lambda^{\mathrm{T}} a_{1}\right) x_{1}^{\mathrm{L}}(\lambda)\right) .
$$

Let us assume that $c$ and $\boldsymbol{a}$ are independent. Then integration of $L(\lambda)$ by parts yields

$$
L(\lambda)=\lambda^{\mathrm{T}} \beta+E \int_{\lambda^{\mathrm{T}} \boldsymbol{a}_{1}}^{\infty}\left(c-\lambda^{\mathrm{T}} \boldsymbol{a}_{1}\right) \mathrm{d} F(c)=\lambda^{\mathrm{T}} \beta+E \int_{\lambda^{\mathrm{T}} \boldsymbol{a}_{1}}^{\infty}(1-F(c)) \mathrm{d} c .
$$

We notice that, under our assumption that $E c_{1}<\infty, L(\lambda)$ is finite for each $\lambda \geqslant 0$. Additional assumptions with respect to the distribution of $c$ and $a$ allow us to establish other smoothness properties of $L(\lambda)$ as shown below. Throughout this section we will assume that $c$ and $\boldsymbol{a}$ are independent and that $E a_{1}$ is finite.

Lemma 4.1. If the distribution function $F$ of $c$ is continuous, then $L(\lambda)$ is differentiable and its gradient is given by

$$
\nabla L(\lambda)=\beta-E \boldsymbol{a}_{1} \boldsymbol{x}_{1}^{\mathrm{L}}(\lambda) .
$$

Proof. Differentiability of $L(\lambda)$ is ensured through Theorem 23.1 in [11, p. 213]. To compute $\partial L / \partial \lambda_{k}(k=1, \ldots, m)$ suppose for the moment that differentiation and integration may be interchanged. Then

$$
\frac{\partial L(\lambda)}{\partial \lambda_{k}}=\beta_{k}-E\left(\left(1-F\left(\lambda^{\mathrm{T}} \boldsymbol{a}_{1}\right)\right) \frac{\partial \lambda^{\mathrm{T}} \boldsymbol{a}_{1}}{\partial \lambda_{k}}\right)=\beta_{k}-E\left(\boldsymbol{a}_{k 1}\left(1-F\left(\lambda^{\mathrm{T}} \boldsymbol{a}_{1}\right)\right),\right.
$$

which, from the independence of $c$ and $a$, is equal to

$$
\frac{\partial L(\lambda)}{\partial \lambda_{k}}=\beta_{k}-E \boldsymbol{a}_{k 1} \boldsymbol{x}_{1}^{\mathrm{L}}(\lambda) \text {. }
$$

Under the assumption that $E a_{1}$ is finite the interchange of expectation and derivative can be justified by the Dominated Convergence Theorem as in Application $3^{\circ}$ of [8, p. 126].

Since the constraints $\lambda \geqslant 0$ satisfy the first-order constraint qualifications in $\lambda^{*}$ [3], Kuhn-Tucker conditions hold at $\lambda^{*}$.

Lemma 4.2. $\lambda^{*}$ satisfies the following conditions for $i=1,2, \ldots, m$ :

(i) $\lambda_{i}^{*}\left(\beta_{i}-E a_{i 1} x_{1}^{\mathrm{L}}\left(\lambda^{*}\right)\right)=0$,

(ii) $E \boldsymbol{a}_{i 1} \boldsymbol{x}_{1}^{\mathrm{L}}\left(\lambda^{*}\right) \leqslant \beta_{i}$. 
Let $f$ be the density function of $c$.

Lemma 4.3. If $F$ is continuous, $f$ is bounded, and $E\left(a_{1} a_{1}^{\mathrm{T}}\right)<\infty$, then $L(\lambda)$ is twice differentiable.

Proof. Differentiation of (4.1) with respect to $\lambda_{l}$ yields

$$
\frac{\partial L(\lambda)}{\partial \lambda_{k} \partial \lambda_{l}}=-\frac{\partial}{\partial \lambda_{l}} E\left(\boldsymbol{a}_{k 1}\left(1-F\left(\lambda^{\mathrm{T}} \boldsymbol{a}_{1}\right)\right)=E\left(\boldsymbol{a}_{k 1} \boldsymbol{a}_{l 1} f\left(\lambda^{\mathrm{T}} \boldsymbol{a}_{1}\right)\right),\right.
$$

since expectations and derivatives may be interchanged under the assumptions of the lemma, which cause the last term of (4.2) to be bounded. Again the interchange is justified by the Dominated Convergence Theorem [8, p. 126].

Lemma 4.4. Assume that $c$ has bounded density function $f$ and that $F$ is continuous, has bounded non-singular support $[\mu, \nu]$, and is strictly increasing over $[\mu, \nu]$. Furthermore assume that the random vector a has a density function with positive density over a convex and open set, and that $E\left(a_{1} a_{1}^{\mathrm{T}}\right)<\infty$. Then $L(\lambda)$ has a unique minimum.

Proof. From Lemma 3.1 we know that $L(\lambda)$ has a minimizer, which we denote by $\lambda^{*}$. Suppose for the moment that there exists a vector $a_{0}$ with positive density such that $\mu<\lambda^{* \mathrm{~T}} a_{0}<\nu$. Then there is a neighbourhood $N_{\lambda}$ of $\lambda^{*}$ such that for any $\lambda \in N_{\lambda}$ : we have $\mu<\lambda^{\mathrm{T}} a_{0}<\nu$. Since the assumptions of Lemma 4.3 are satisfied, equation (4.2) holds. Moreover, since $F$ is strictly increasing over $[\mu, \nu], f$ is positive almost everywhere over $[\mu, \nu]$ so that for all $\lambda \in N_{\lambda^{*}}$ the second order derivative matrix of $L(\lambda)$ is positive definite. Therefore $\lambda^{*}$ is a unique local minimum and by the convexity of $L(\lambda)$ also the unique global minimum.

We show that a vector $a_{0}$ exists for which $\mu<\lambda^{*} a_{0}<\nu$. First suppose that no such vector exists, then either $\lambda^{* \mathrm{~T}} a>\nu$ for all $a$ with positive density or $\lambda^{* \mathrm{~T}} a<\mu$ for all $a$ with positive density (since this set is open and convex). In the former case $L(\lambda)=\lambda^{\mathrm{T}} \beta$ for all $\lambda$ in some neighbourhood of $\lambda^{*}$. Since $\lambda^{* \mathrm{~T}} a>\nu$ implies that $\lambda^{*} \neq 0$ it is easy to see that by decreasing positive components of $\lambda^{*}$ we obtain a function value of $L(\lambda)$ smaller than $L\left(\lambda^{*}\right)$. In the other case, $\lambda^{* \mathrm{~T}} a<\mu$, we have that $L(\lambda)=\lambda^{\mathrm{T}} \beta+E c_{1}-\lambda^{\mathrm{T}} E a_{1}$ for all $\lambda$ in some neighbourhood of $\lambda^{*}$. Since we assumed that $\beta<E \boldsymbol{a}_{1}, L(\lambda)$ can be decreased below $L\left(\lambda^{*}\right)$ by increasing $\lambda^{*}$, again providing a contradiction. Thus, there exists a vector $a_{0}^{\prime}$ with positive density such that $\mu \leqslant \lambda^{* \mathrm{~T}} a_{0}^{\prime} \leqslant \nu$. By the assumption that the set on which the density of $\boldsymbol{a}$ is positive is open and convex there also exists a vector $a_{0}$ with positive density such that $\mu<\lambda^{* \mathrm{~T}} a_{0}<\nu$, which completes the proof.

\section{Special cases: $m=1, m=2$}

In this section, we assume (as in $[4,7,10]$ ), that the profit coefficients $c_{j}$ as well as the requirements $a_{i j}$ are independent uniformly distributed over $[0,1], j=1, \ldots, n$, $i=1, \ldots, m$, and carry out some actual computations on the solution value of $\mathrm{MK}$. 
In the case that $m=1$, straightforward calculations lead to the following formulae:

$$
\begin{aligned}
& \operatorname{Eax}^{\mathbf{L}}(\lambda)=\left\{\begin{array}{ll}
\frac{1}{2}-\frac{1}{3} \lambda & \text { if } \lambda \leqslant 1, \\
1 /\left(6 \lambda^{2}\right) & \text { if } \lambda>1,
\end{array} \quad \operatorname{Ec} x^{\mathbf{L}}(\lambda)= \begin{cases}\frac{1}{2}-\frac{1}{6} \lambda^{2} & \text { if } \lambda \leqslant 1, \\
1 /(3 \lambda) & \text { if } \lambda>1,\end{cases} \right. \\
& L(\lambda)= \begin{cases}\lambda\left(\beta-\frac{1}{2}+\frac{1}{3} \lambda\right)+\frac{1}{2}-\frac{1}{6} \lambda^{2} & \text { if } \lambda \leqslant 1, \\
\lambda\left(\beta-1 /\left(6 \lambda^{2}\right)\right)+1 /(3 \lambda) & \text { if } \lambda>1,\end{cases} \\
& \lambda^{*}= \begin{cases}(6 \beta)^{-1 / 2} & \text { if } 0<\beta<\frac{1}{6}, \\
\frac{3}{2}-3 \beta & \text { if } \frac{1}{6} \leqslant \beta<\frac{1}{2},\end{cases} \\
& L\left(\lambda^{*}\right)= \begin{cases}\left(\frac{2}{3} \beta\right)^{1 / 2} & \text { if } 0<\beta<\frac{1}{6}, \\
-\frac{3}{2} \beta^{2}+\frac{3}{2} \beta+\frac{1}{8} & \text { if } \frac{1}{6} \leqslant \beta<\frac{1}{2} .\end{cases}
\end{aligned}
$$

The graph of $L\left(\lambda^{*}\right)$ as a function of $\beta$ is shown in Figure 1. Notice that in accordance with Lemmas 4.1 and $4.2, \operatorname{Eax}_{1}^{\mathrm{L}}\left(\lambda^{*}\right)-\beta=0$.

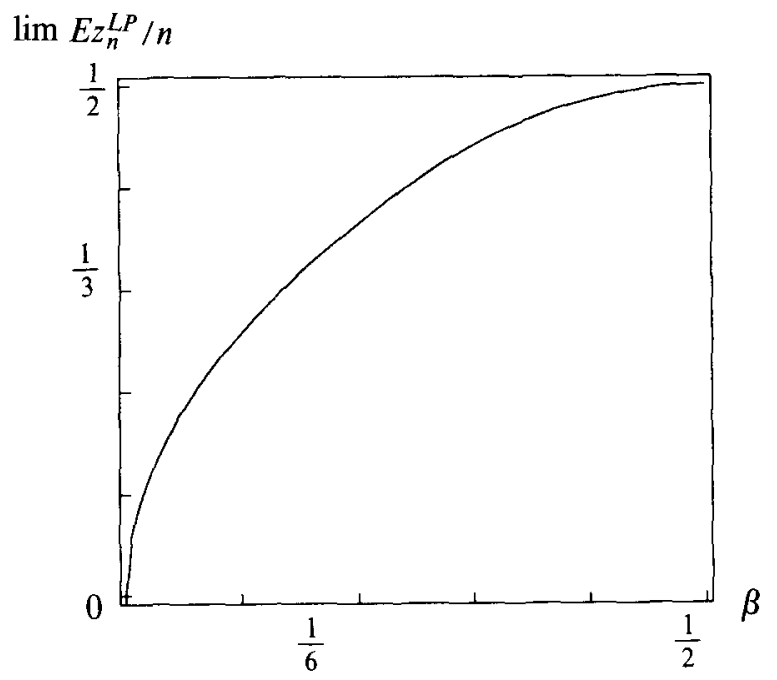

Fig. 1.

In the case that $m=2, E c x_{1}^{\mathrm{L}}(\lambda), E \boldsymbol{a}_{1} \boldsymbol{x}_{1}^{\mathrm{L}}(\lambda)$ and $E \boldsymbol{a}_{2} \boldsymbol{x}_{1}^{\mathrm{L}}(\lambda)$ take different forms over different regions. If $\beta_{1} \geqq \beta_{2}$, these are defined as follows:

$$
\begin{aligned}
& A:=\left\{\left(\beta_{1}, \beta_{2}\right) \mid \beta_{1} \geqslant \beta_{2}, \beta_{2} \geqslant 24 \beta_{1}^{2}\right\}, \\
& B:=\left\{\left(\beta_{1}, \beta_{2}\right) \mid \beta_{2} \leqslant \frac{8}{3} \beta_{1}^{2}, \beta_{2} \leqslant \frac{1}{6}, \beta_{1}<\frac{1}{2}\right\}, \\
& C:=\left\{\left(\beta_{1}, \beta_{2}\right) \mid \beta_{2} \leqslant \frac{4}{3} \beta_{1}-\frac{1}{6}, \beta_{2}>\frac{1}{6}, \beta_{1}<\frac{1}{2}\right\}, \\
& D:=\left\{\left(\beta_{1}, \beta_{2}\right) \mid \beta_{1} \geqslant \beta_{2}, \beta_{2}>\frac{4}{3} \beta_{1}-\frac{1}{6}, \beta_{1}+\beta_{2} \geqslant \frac{5}{12}\right\}, \\
& E:=\left\{\left(\beta_{1}, \beta_{2}\right) \mid \beta_{1} \geqslant \beta_{2}, \beta_{2}>\frac{8}{3} \beta_{1}^{2}, \beta_{1}+\beta_{2}<\frac{5}{12}\right\} .
\end{aligned}
$$

The regions are depicted in Figure 2. 


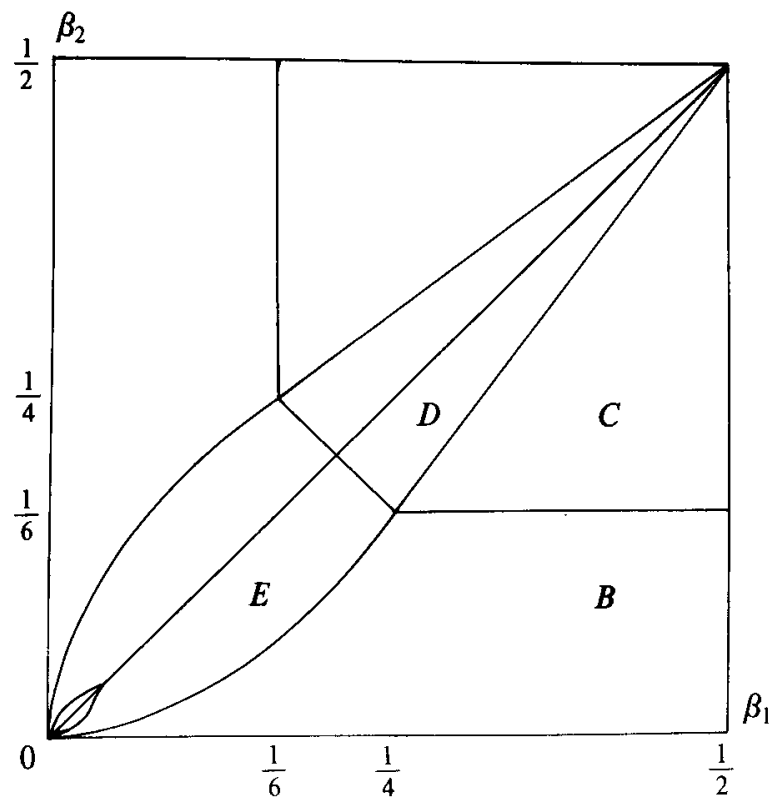

Fig. 2.

The values of $\lambda^{*}$ and $L\left(\lambda^{*}\right)$ in the corresponding five regions where $\beta_{1}<\beta_{2}$ can be obtained by exchanging $\beta_{1}$ with $\beta_{2}$ and $\lambda_{1}$ with $\lambda_{2}$ in the formulae given below.

$$
\begin{array}{ll}
\text { Region A: } & \lambda_{1}^{*}=\left(\beta_{2} /\left(24 \beta_{1}^{2}\right)\right)^{1 / 3}, \lambda_{2}^{*}=\left(\beta_{1} /\left(24 \beta_{2}^{2}\right)\right)^{1 / 3}, \\
& L\left(\lambda^{*}\right)={ }_{2}^{1}\left(9 \beta_{1} \beta_{2}\right)^{1 / 3} ; \\
\text { Region B: } & \lambda_{1}^{*}=0, \lambda_{2}^{*}=\left(1 /\left(6 \beta_{2}\right)\right)^{1 / 2}, L\left(\lambda^{*}\right)=\left({ }_{3}^{2} \beta_{2}\right)^{1 / 2} ; \\
\text { Region C: } & \lambda_{1}^{*}=0, \lambda_{2}^{*}={ }_{2}^{3}-3 \beta_{2}, L\left(\lambda^{*}\right)=\frac{3}{2} \beta_{2}^{2}+\frac{3}{2} \beta_{2}+\frac{1}{8} ; \\
\text { Region D: } & \lambda_{1}^{*}=\frac{1}{7}\left(36 \beta_{2}-48 \beta_{1}+6\right), \lambda_{2}^{*}=\frac{1}{7}\left(36 \beta_{1}-48 \beta_{2}+6\right), \\
& L\left(\lambda^{*}\right)=\frac{1}{7}\left(-24 \beta_{2}^{2}+36 \beta_{1} \beta_{2}+6 \beta_{2}+\frac{1}{2}\right) .
\end{array}
$$

Region E: A closed form equation for the values $\lambda^{*}, L\left(\lambda^{*}\right)$ with $\beta$ lying in $E$ is complicated, though not impossible, since it involves the solution of an equation of degree four. Numerical evaluation is easier through use of an appropriate non-linear programming routine.

A picture of the surface $L\left(\lambda^{*}\right)$, defined over the $\left(\beta_{1}, \beta_{2}\right)$ plane and evaluated either analytically or numerically, is presented in Figure 3.

Calculation of $\beta_{i}-E a_{i 1} x_{1}^{\mathrm{L}}\left(\lambda^{*}\right)(i=1,2)$ for the regions $\mathrm{A}, \mathrm{B}, \mathrm{C}$ and $\mathrm{D}$ yields the following result. In $A$ and $D$ both $\beta_{1}-E a_{11} x_{1}^{\mathrm{L}}\left(\lambda^{*}\right)=0$ and $\beta_{2}-E a_{21} x_{1}^{\mathrm{L}}\left(\lambda^{*}\right)=0$, while in $\mathrm{B}$ and $\mathrm{C}$ only $\beta_{2}-E \boldsymbol{a}_{21} \boldsymbol{x}_{1}^{\mathrm{L}}\left(\lambda^{*}\right)=0$ and $\beta_{1}-E \boldsymbol{a}_{11} \boldsymbol{x}_{1}^{\mathrm{L}}\left(\lambda^{*}\right)>0$. This says that in $\mathrm{A}$ and $D$ the expected slack in both constraints tend to 0 in an optimal solution, while in $\mathrm{B}$ and $\mathrm{C}$ this is only true for the tighter constraint. This corresponds to intuition: when $\beta_{2}$ is sufficiently small with respect to $\beta_{1}$, as in $\mathrm{B}$ and $\mathrm{C}$, the first constraint 


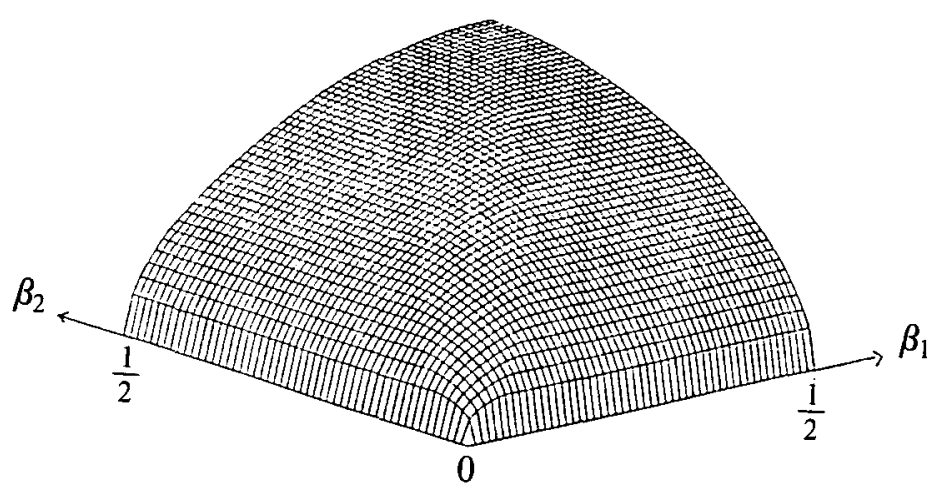

Fig. 3.

can be disregarded and MK is reduced to a single knapsack problem. To support this conclusion, observe that the values of $\lambda_{2}^{*}$ and $L\left(\lambda^{*}\right)$ obtained for the regions $\mathrm{B}$ and $\mathrm{C}$ are identical to the corresponding ones derived for the case $m=1$.

Similar calculations can be carried out for $m \geqslant 3$, even though in these cases only numerical approximation of $\lambda^{*}$ and $L\left(\lambda^{*}\right)$ is possible for many values of $\beta$. The computation of $L(\lambda)$ and $\nabla L(\lambda)$ amounts to integrating the density function over regions defined by linear inequalities. In many situations, closed form expressions for these integrals can be derived in principle.

The implication of the results presented for the analysis of certain generalized greedy solution methods for MK will form the subject of a future paper.

\section{Acknowledgement}

We gratefully acknowledge the useful comments of an anonymous referee on an earlier version of this paper, which led to a considerable improvement in the exposition.

\section{References}

[1] G. d'Atri, "Probabilistic analysis of the knapsack problem," Technical Report No. 7, Groupe de Recherche 22, Centre National de la Recherche Scientifique (Paris, 1978).

[2] G. Ausiello, A. Marchetti-Spaccamela and M. Protasi, "Probabilistic analysis of the solution of the knapsack problem," in: Proceedings of the Tenth IFIP Conference (Springer, New York, 1982) pp. 557-565.

[3] A.V. Fiacco and G.P. McCormick, Nonlinear Programming: Sequential Unconstrained Minimization Techniques (Wiley, New York, 1968).

[4] A.M. Frieze and M.R.B. Clarke, "Approximation algorithms for the $m$-dimensional $0-1$ knapsack problem: worst-case and probabilistic analysis," European Journal of Operations Research 15 (1984) 100-109.

[5] M.R. Garey and D.S. Johnson, "Strong NP-completeness results: motivation, examples and implications," Journal of the Association of Computer Machinery 25 (1978) 499-508. 
[6] M.R. Garey and D.S. Johnson, Computers and Intractability: A Guide to the Theory of NPCompleteness (Freeman, San Francisco, CA, 1979).

[7] A.V. Goldberg and A. Marchetti-Spaccamela, "On finding the exact solution of a $0-1$ knapsack problem," in: Proceedings of the 16th ACM Symposium on Theory of Computing (Association for Computing Machinery, New York, 1984) pp. 359-368.

[8] M. Loève, Probability Theory (Springer, New York, 1977).

[9] J. Lorie and L. Savage, "Three problems in capital rationing," Journal of Business 28 (1955) 229-239.

[10] G.S. Lueker, "On the average difference between the solution to linear and integer knapsack problems," Applied Probability-Computer Science, the Interface, Vol. 1 (Birkhauser, Basel, 1982) pp. 489-504.

[11] R.T. Rockafellar, Convex Analysis (Princeton University Press, Princeton, NJ, 1970).

[12] B. Weide, "Statistical methods in algorithm design and analysis," Ph-D thesis, CMU-CS 78-142, Carnegie-Mellon University (Pittsburgh, PA, 1978). 